\title{
Percepciones sobre la diversidad en La Matanza. Reflexiones a partir de la aplicación de una encuesta
}

\section{Perceptions of diversity in La Matanza. Reflections on the implementation of a survey}

Yamila Soledad Abal yamila.abal@gmail.com CONICET - Instituto Multidisciplinario de Historia y Ciencias Humanas, Argentina

Cecilia Eleonora Melella cemelella@gmail.com CONICET - Instituto de Desarrollo Económico y Social / Facultad de Ciencias Sociales - Instituto de Investigaciones Gino Germani. Universidad de Buenos Aires, Argentina

Brenda Matossian bmatossian@gmail.com CONICET - Instituto Multidisciplinario de Historia y Ciencias Humanas, Argentina

Recepción: 01 Diciembre 2020

Aprobación: 01 Junio 2021

Publicación: 02 Agosto 2021

Cita sugerida: Abal, Y. S., Melella, C. E. y Matossian, B. (2021). Percepciones sobre la diversidad en La Matanza. Reflexiones a partir de la aplicación de una encuesta. Cuestiones de Sociología, 25, e123. https://doi.org/10.24215/23468904e123
Resumen: El objetivo del artículo es identificar la percepción sobre la diversidad cultural entre distintos tipos de actores en La Matanza. Se analizan las concepciones sobre diversidad cultural que subyacen a las diferentes políticas públicas a escala local y se indaga en las representaciones que construye la población general respecto de las migraciones y de la diversidad cultural en el municipio. La estrategia metodológica se basó en la triangulación de fuentes, promoviendo el diálogo entre resultados de una encuesta multi-situada con fuentes censales y el relevamiento de políticas públicas dedicadas a la diversidad. Como resultados, se identifica una concepción monolítica de la cultura que la asocia con atributos preexistentes y estancos como la nación y la etnia. También se reconoce que las políticas públicas vinculadas con la diversidad en el municipio han ido en aumento. Sobre la percepción de la diversidad cultural en la población, se registra un sobredimensionamiento hacia la comunidad boliviana y la persistencia de representaciones de carácter folklorizante que se construyen sobre la posesión de rasgos esenciales manifestados en la gastronomía, las festividades y las lenguas.

Palabras clave: Migraciones, Diversidad cultural, Políticas públicas, La Matanza.

Abstract: The objective of this article is to identify the perception of cultural diversity among different types of actors in La Matanza. The conceptions about cultural diversity that underlie the different public policies at the local scale are analyzed and the representations that general population constructs regarding migrations and cultural diversity in the district are investigated. The methodological strategy was based on the triangulation of sources, promoting dialogue between the results of a multi-sited survey with census sources and the gathering of public policies dedicated to diversity. As results, a monolithic conception of culture is identified, which associates it with pre-existing and rigid attributes such as nation and ethnicity; moreover public policies related to diversity in the district have been increasing. Regarding the perception of cultural diversity of the population, there is an over-dimensioning towards the Bolivian community and a persistence of folkloric character representations built upon the possession of essential features manifested in gastronomy, festivities and languages.

Keywords: Migrations, Cultural diversity, Public policies, La Matanza. 


\section{1- Introducción}

La diversidad cultural se ha constituido como una temática de amplio interés en su articulación con los procesos migratorios que se dan en todo el mundo y que desafían todas las escalas de gobierno, especialmente en los países cuya población contiene proporciones importantes de nacidos/as fuera de la Argentina. Las políticas públicas focalizadas en la diversidad cultural tienden a ser analizadas a escalas de los Estados nacionales, mientras que las escalas locales, especialmente en áreas alejadas de los centros de poder, han recibido una menor atención.

Este trabajo, enmarcado en un proyecto de investigación mayor, ${ }^{1}$ tiene como objetivo identificar y explicar, desde el entramado multi-actoral, la percepción de la diversidad cultural entre actores públicos y privados que intervienen con sus acciones y prácticas sociales para la implementación territorial de los programas de inclusión social y la interculturalidad a escala local. Este análisis se halla acotado al partido de La Matanza, municipio de gran concentración de población de la provincia de Buenos Aires, como se especificará en el desarrollo. Más específicamente, este artículo atraviesa dos dimensiones de análisis respecto de la diversidad. Por un lado, indaga sobre las definiciones latentes acerca de la diversidad (cultural), recuperando algunos de los debates más representativos sobre su gestión desde las esferas estatales. También se referencian las principales políticas públicas sobre la diversidad que se administran en el municipio de La Matanza. Por otro lado, se concentra en el análisis de las percepciones que tienen los diferentes actores sociales acerca de la diversidad cultural, la inclusión social, el acceso a los derechos y la conformación de un escenario intercultural en el mismo partido.

En este sentido, asentamos el trabajo sobre los supuestos de que las políticas públicas para la promoción de la diversidad cultural y del diálogo intercultural de los/las migrantes no son homogéneas: algunas tenderían a la mera inclusión y otras no los tienen en cuenta. Paralelamente, existen acciones y prácticas sociales relacionadas con procesos y espacios interculturales, donde los/las migrantes devienen en actores nodales para llevar adelante la implementación de esas políticas públicas en sus distintos niveles de gestión. Entendemos que las percepciones sobre la diversidad resultan nodales a la hora de plantear una articulación verdaderamente intercultural entre la esfera estatal y las esferas de la sociedad civil, en particular los/ las migrantes, que propicie la participación y la potencia política de estos sujetos y grupos.

Este artículo se estructura en siete apartados. Luego de esta Introducción, en el apartado 2, "La diversidad cultural en debate", se recuperan brevemente algunas discusiones dentro del campo social respecto del concepto de diversidad cultural, caracterizadas por la tensión entre universalidad y particularidad. La sección 3 es estrictamente metodológica y se concentra en la descripción de la encuesta utilizada para llevar a cabo esta investigación. El apartado 4, "Caracterización de La Matanza y su diversidad migratoria desde la mirada geodemográfica. 
Generalidades y particularidades", tiene como objetivo ofrecer un panorama sobre la heterogeneidad que identifica a este municipio, haciendo foco en la cuestión migratoria. La parte 5, "Las representaciones desde el Estado a escala municipal. Hacia una mayor complejización de la diversidad”, analiza las políticas y programas de gestión estatal, focalizando en las migraciones internacionales. El apartado 6 presenta el nodo de este artículo al exponer parte de los resultados de la encuesta. Si bien esta abordó también dimensiones como las percepciones sobre el municipio de La Matanza y sobre las principales problemáticas que afectan a la comunidad, o las representaciones en relación con el reconocimiento y acceso a derechos de la población migrante y el uso de medios de comunicación, en este trabajo nos enfocaremos en el análisis de la información que la encuesta relevó específicamente sobre las representaciones sobre migraciones y diversidad cultural. Por último, las reflexiones finales presentan algunas conclusiones y abren nuevos interrogantes.

\section{2- La diversidad cultural en debate}

La diversidad cultural es un término complejo y polisémico que deviene necesariamente contextual. El modo como se administra la diversidad cultural y se promueven (o no) las relaciones interculturales en instituciones y/o a través de las políticas depende de un modo u otro de las condiciones económicas y políticas a nivel local, nacional e internacional, así como del poder de las instituciones para definir y naturalizar (desde arriba) y de los conflictos y negociaciones que tienen lugar en las prácticas (desde abajo). Por consiguiente, como sostiene Susan Wright (1998), pone en evidencia la centralidad de concebir la cultura en su dimensión de politicidad. Si hacemos un poco de historia, a partir de la segunda posguerra mundial la cultura comienza a estar presente en la agenda estatal, pero es en la década de 1990 que se producen transformaciones a escala en el escenario mundial sobre la diferencia cultural y el reconocimiento, que fueron introducidas en el campo político y en el de los tomadores de decisiones en una gama de territorios de poder. Sin embargo, esta inclusión pretendió ocultar la politicidad que el mismo concepto de cultura implica. Wright (1998) plantea la existencia de dos significados de cultura: uno viejo y uno nuevo. El primero refiere a ideas y significados definidos e inamovibles que son compartidos por toda la población, compuesta por individuos homogéneos (idea de cultura auténtica). El segundo surge hacia finales de la década de 1970 y entiende la cultura como proceso activo de construcción de significaciones que incluye la disputa por su misma definición, que se encuentra atravesada por los procesos históricos. Es decir, sólo la forma hegemónica de la cultura aparece como coherente, sistemática y consensual.

En esta línea interpretativa, Sara Ahmed (2007) plantea que el concepto de diversidad también reemplazó a otras conceptualizaciones, como equidad y justicia social, que fracasaron por la imposibilidad de las mismas instituciones para llevarlas a cabo. Así, la diversidad -en tanto significante vacío- conlleva en sí mismo la lucha por definir su significación, pues es un término que se usa estratégicamente e implica negociaciones y resistencias sobre qué significa para el statu quo y qué puede significar en pos de la lucha contra la inequidad social. Resulta nodal establecer quién define la diversidad y, especialmente, prestar atención a los procesos que tienen lugar cuando ésta se pone en acción 
en el universo de las prácticas y de los/las sujetos/as involucrados/as. Aun así, como señala Ahmed, el peligro reside en que "decir diversidad no es hacer diversidad” (Ahmed, 2007, p. 254) pues, por sí misma, la diversidad no es algo simple de llevar a cabo.

Asimismo, la diversidad cultural retoma y complejiza la relación entre la universalidad y la particularidad al plantear la aparente aporía entre la abolición de la diferenciación y la valorización de la especificidad. Nancy Fraser (2000), en uno de los escritos más categóricos sobre la temática, sostiene que los gobiernos occidentales han dado respuestas afirmativas ante esta cuestión al promover una noción de justicia económica asociada al Estado liberal benefactor que pretende reparar la mala distribución de los recursos dejando intacta la estructura político-cultural subyacente. La crítica de la autora establece que estas soluciones mantienen las diferencias y ayudan a profundizarlas (por ejemplo, entre trabajadores activos y desocupados); es decir, pese al objetivo de resolver injusticias de redistribución, se terminan generando injusticias de reconocimiento. En el plano cultural, se promueven políticas afirmativas del multiculturalismo central que proponen la revaluación de identidades a partir de la diferenciación de grupos sobre una cultura fosilizada que establece una diversidad pasteurizada (Briones, 2007). Las soluciones afirmativas incluyen una contradicción interna porque promueven una concepción universalista del reconocimiento, pero ponen en práctica una dinámica estigmatizante de este. Por su parte, las soluciones transformadoras que promueve la autora combinan los programas universales de bienestar social pero con el objetivo de socavar, a través de una reestructuración profunda de las relaciones de producción, las diferenciaciones de clase. Pretenden, desde una propuesta socialista, difuminar los factores de diferenciación entre los grupos y tienden a promover reciprocidad y solidaridad en las relaciones de reconocimiento. Respecto de las injusticias culturales, se asocian a la deconstrucción al plantear que la eliminación de la falta de respeto se lograría mediante la transformación de la estructura culturalvalorativa subyacente y esto desestabilizaría la diferenciación entre grupos.

\section{3 - Estrategia metodológica}

En primer lugar, se contempló la realización de encuestas que combinasen el tipo exploratorio y el descriptivo, y permitieran documentar las condiciones de los actores en un momento determinado. Se realizaron encuestas multi-situadas (Padilla, Azevedo y Olmos-Alcaraz, 2014) y para su implementación se configuró una muestra no probabilística e intencional (o por conveniencia), compuesta por 201 personas adultas que residieran en el partido de La Matanza.

Con el objetivo de identificar heterogeneidades en el interior del partido de La Matanza y entre las mismas localidades, se seleccionaron cinco puntos muestrales dispersos en diferentes localidades del partido, pero con características similares para garantizar cierto grado de homogeneidad y tornar comparables las respuestas obtenidas. Para ello, las encuestas en los diferentes puntos fueron tomadas en similares condiciones: se las implementó un día hábil de 16 a $18 \mathrm{~h}$ y se buscó tomar cuotas similares de personas encuestadas según localidad (aproximadamente, 40 por punto muestral).Se definieron como puntos muestrales las plazas ubicadas en las áreas comerciales cercanas (a no más de cinco cuadras) de las estaciones 
ferroviarias de cinco localidades del partido: San Justo, Ramos Mejía, Tapiales, Gregorio de Laferrere y González Catán. Esta selección se fundamenta a partir de la inserción de estos puntos en áreas que corresponden a lo que Abba (2005) denomina "centralidades urbanas tradicionales". Se trata de áreas, configuradas y ramificadas sobre la red ferroviaria y alrededor de las estaciones, que cumplen el rol de estructurar las dinámicas de la región metropolitana y canalizar los mayores volúmenes de los flujos de personas. El trabajo de campo tuvo lugar entre el 16 de agosto y el 13 de septiembre de 2019. Se utilizó un cuestionario semi-estructurado previamente diseñado, compuesto por preguntas cerradas y abiertas, agrupadas según ejes temáticos. Uno de ellos, percepciones sobre migraciones y diversidad cultural, es el trabajado en este artículo.

Además de las fuentes primarias producidas a partir de la encuesta, se utilizaron fuentes secundarias con el propósito de contraponer los resultados obtenidos con otros tipos de análisis. Por un lado, se contrastaron las respuestas con fuentes censales y, desde otra perspectiva, se complementaron los resultados del propio relevamiento con las políticas públicas identificadas a escala municipal dedicadas a la diversidad.

Para el diálogo con fuentes censales se indagó el Censo Nacional de Población, Hogares y Viviendas 2010 y, en menor medida, el 2001, a escala del conjunto del municipio para identificar cambios en la composición migratoria matancera. Asimismo, a partir de agregar microdatos censales del 2010 a escala de radios, se reconstruyeron las particularidades de cada una de las localidades que conforman el partido con el objetivo de describir y examinar heterogeneidades en su interior. El análisis de las variables censales referidas a lugar y país de nacimiento, a lo largo del tiempo y del espacio, permitió indagar en los procesos migratorios y reconocer la variación espacial de la distribución de la población migrante en el interior del municipio. Más allá de las diferencias que impiden pensar en términos de comparabilidad lineal, dadas las brechas temporales y las disimilitudes en los tipos de relevamientos, se aspira a tener evidencias lo más recientes posible para contrastar las percepciones de la población sobre las migraciones con los registros más confiables y actualizados disponibles.

Por su parte, el análisis de las políticas públicas municipales sobre la diversidad fue abordado desde una metodología cualitativa a través del análisis de contenido de normativa, programas y proyectos. Se indagaron páginas Web oficiales y de funcionarios/as de relevancia, así como de las redes sociales (Facebook e Instagram) a partir de la identificación de menciones sobre "diversidad", "cultura", "interculturalidad", "multiculturalidad", "identidad" y "migración", tanto en lenguaje escrito como visual, con el objetivo de armar el corpus. También, se realizaron entrevistas en profundidad a funcionarios/as municipales y/o informantes clave. Por último, se consultaron algunas notas periodísticas relevantes sobre la temática, en especial de medios locales. A partir de este corpus de análisis se pasó a una fase interpretativa, que opera principalmente en el nivel pragmático y que se estructuró sobre la aproximación entre los componentes del discurso y las condiciones sociales, institucionales, ideológicas, culturales o históricas en las que fueron enunciados. 


\section{4 - Caracterización de La Matanza y su diversidad migratoria desde la mirada geodemográfica. Generalidades y particularidades dentro del partido}

La Región Metropolitana de Buenos Aires (RMBA) es uno de los espacios que más fuerte atracción ha ejercido para la migración internacional en la Argentina. En 2010, los extranjeros ${ }^{2}$ representaban el 8,33\% del total de la población mientras que la media nacional era de 4,5\%. Dentro de sus jurisdicciones se destacan La Matanza, Lanús, Lomas de Zamora y General San Martín con más de un 9\% de extranjeros (Sassone y Matossian, 2014)). Para el caso de La Matanza, el porcentaje de nacidos/as en países distintos de la Argentina se incrementó en el último período intercensal: más precisamente, del 8,67\% en 2001 al 9,67\% para el 2010. Estos datos dan cuenta de la relevancia que el componente migratorio tiene en la población de este municipio.

Más allá de estos datos generales, interesa avanzar hacia una mirada que contemple la diversidad dentro de estos conjuntos que suelen ser considerados como homogéneos. Para ello, se comparan los datos por país de nacimiento de la población extranjera en los dos últimos registros censales, 2001 y 2010, en La Matanza (Tabla 1). Así, respecto de los principales orígenes de los/las migrantes internacionales que residen en el partido se identifica que en los dos últimos censos se repiten los tres primeros lugares en orden de importancia: Paraguay, Bolivia e Italia, aunque ha cambiado el protagonismo decada uno. Los/ as nacidos/as en Paraguay han aumentado su importancia no sólo en términos absolutos sino también relativos, pues su participación se incrementó de un 36\% a un $45 \%$, casi la mitad del total de los/las no nativos/as. En segundo lugar, la población nacida en Bolivia ha duplicado su cuantía entre 2001 y 2010, y agrupa casi un $28 \%$ del total de los/las extranjeros/as. En sumatoria, estos dos países vecinos representan casi tres cuartos del total de migrantes internacionales. Con una tendencia opuesta, para el caso de nacidos/as en Italia, es notable el descenso de su importancia: de casi el 20\% en 2001 pasó a representar poco más del 9\% en 2010. Al igual que ocurre en el total del país y en buena parte de las ciudades argentinas, la población nacida en Perú se ha sumado con creciente protagonismo al grupo de nacidos/as en países sudamericanos. En el caso de La Matanza, ha pasado de ocupar el séptimo lugar en importancia a principios del siglo XXI a alcanzar el cuarto en el último censo. En términos absolutos, la población peruana se ha triplicado en este período. En contraposición, los/las nacidos/as en España han descendido a la mitad en su porcentaje de representatividad. Por último, cabe señalar el caso de la población nacida en Portugal, cuyo protagonismo histórico en el partido ha sido destacado por su rol en el desarrollo de fábricas de ladrillos y en la producción de hortalizas y verduras durante la primera mitad del siglo XX (Svetlitza de Nemirovsky y Gonzalez, 1999). Esta migración europea se muestra, como en los casos de la italiana y la española, también en descenso en el último período intercensal, pues ha perdido peso: del $1 \%$ al $0,44 \%$ del total de la población nacida en países distintos de la Argentina. 
Tabla 1

Población extranjera por país de nacimiento, La Matanza 2001-2010

\begin{tabular}{|l|l|r|r|l|r|r|}
\hline & País de Nacimiento 2001 & Total 2001 & \% & Pais de Nacimiento 2010 & Total 2010 & \% \\
\hline 1 & Paraguay & 39.193 & 36,03 & Paraguay & 77.807 & 45,32 \\
\hline 2 & Bolivia & 21.875 & 20,11 & Bolivia & 47.932 & 27,92 \\
\hline 3 & Italia & 21.712 & 19,96 & Italia & 16.098 & 9,38 \\
\hline 4 & España & 7.068 & 6,50 & Perú & 8.092 & 4,71 \\
\hline 5 & Uruguay & 6.476 & 5,95 & Uruguay & 7.660 & 4,46 \\
\hline 6 & Chile & 3.104 & 2,85 & España & 5.269 & 3,07 \\
\hline 7 & Perú & 2.195 & 2,02 & Chile & 3.787 & 2,21 \\
\hline 8 & Portugal & 1.089 & 1,00 & Brasil & 890 & 0,52 \\
\hline 9 & Brasil & 630 & 0,58 & Portugal & 751 & 0,44 \\
\hline 10 & Polonia & 450 & 0,41 & China & 423 & 0,25 \\
\hline & Total extranjeros & 108.783 & 100 & Total extranjeros & 171.682 & 100 \\
\hline & Total argentinos & 1.146 .505 & & Total argentinos & 1.604 .134 & 10,70 \\
\hline & \% Extranjeros / Total & 9,49 & & \% Extranjeros / Total & & \\
\hline
\end{tabular}

Fuente: Elaboración propia sobre la base de información del INDEC 2001 y 2010

En síntesis, la transformación en el perfil migratorio, de un protagonismo mayormente europeo a uno cada vez más sudamericano, se ha ido registrando lentamente en todo el país, modificando el conjunto de población extranjera, desde el censo de 1947 (Devoto, 2004; Pacecca y Courtis, 2008; Sassone y Matossian, 2014, entre muchos otros). La Matanza no ha sido la excepción.

Distribución por país de nacimiento y por localidad: la diversidad al interior matancero

Recorreremos la espacialidad por orígenes para identificar la distribución de la población nacida en los cuatro países con mayor presencia dentro de los/las extranjeros/as (Paraguay, Bolivia, Italia y Perú), cada uno con las singularidades de su variabilidad espacial. La Tabla 2 sintetiza indicadores para las mismas cuatro nacionalidades respecto de las localidades matanceras en las que se aplicó la encuesta. ${ }^{3}$ Se utilizan dos indicadores: uno es el peso demográfico de la población de un determinado origen en las localidades con respecto a su total dentro del partido (\%PLoc/TPLM); el otro es el porcentaje de esa población de referencia con respecto a los/las extranjeros/as en cada una de las localidades (\%PLoc/ PTELoc). De este modo se pretende un diálogo escalar entre el partido y sus localidades.

Para el caso de la colectividad paraguaya, las mayores concentraciones se registran en las localidades más alejadas del límite del partido con la Ciudad Autónoma de Buenos Aires, como es González Catán. A su vez, en esta localidad hay una composición de su población extranjera en la que los/las paraguayos/as tienen un protagonismo en torno al $70 \%$ sobre dicho conjunto, muy superior al del total del partido (45\%). Con respecto a la distribución de la población nacida en Bolivia, resaltan dos concentraciones importantes: una de ellas en el extremo noreste del partido, representado por la localidad de Tapiales, y la segunda en 
G. de Laferrere. Allí el peso de los/las nacidos/as en Bolivia sobre el total de extranjeros/as es superior al que se presenta para el total del partido.

Tabla 2

Localidades de La Matanza en las que fue implementada la Encuesta. Población nacida en Paraguay, Bolivia, Italia y Perú, valores absolutos y relativos, $2010^{4}$

\begin{tabular}{|l|r|r|r|r|r|r|}
\hline & \multicolumn{1}{|c|}{$\begin{array}{c}\text { González } \\
\text { Catán }\end{array}$} & $\begin{array}{c}\text { Gregorio de } \\
\text { Laferrere }\end{array}$ & $\begin{array}{c}\text { Ramos } \\
\text { Mejía }\end{array}$ & $\begin{array}{c}\text { San } \\
\text { Justo }\end{array}$ & Tapiales & \multicolumn{1}{c|}{$\begin{array}{c}\text { Total La } \\
\text { Matanza }\end{array}$} \\
\hline TP Paraguay (PPa) & 14071 & 9921 & 1480 & 5478 & 931 & 77807 \\
\hline \%PPaLoc/TPPaLM & 18,08 & 12,75 & 1,90 & 7,04 & 1,20 & $\mathbf{1 0 0 , 0 0}$ \\
\hline \%PPaLoc/PTELoc & $\mathbf{7 0 , 2 5}$ & 43,23 & 17,64 & 45,60 & 19,12 & $\mathbf{4 5 , 3 2}$ \\
\hline TP Bolivia (PBo) & 3500 & 9574 & 790 & 1538 & 2098 & $\mathbf{4 7 9 3 2}$ \\
\hline \%PBoLoc/TPBoLM & 7,30 & 19,97 & 1,65 & 3,21 & 4,38 & $\mathbf{1 0 0 , 0 0}$ \\
\hline \%PBoLoc/PTELoc & 17,47 & $\mathbf{4 1 , 7 1}$ & 9,41 & 12,80 & $\mathbf{4 3 , 0 8}$ & $\mathbf{2 7 , 9 2}$ \\
\hline TP Italia (PIt) & 231 & 803 & 2680 & 2555 & 318 & $\mathbf{1 6 0 9 8}$ \\
\hline \%PItLoc/TPItLM & 1,43 & 4,99 & 16,65 & 15,87 & 1,98 & $\mathbf{1 0 0 , 0 0}$ \\
\hline \%PItLoc/PTELoc & 1,15 & 3,50 & $\mathbf{3 1 , 9 4}$ & $\mathbf{2 1 , 2 7}$ & 6,53 & $\mathbf{9 , 3 8}$ \\
\hline TP Perú (PPe) & 372 & 523 & 404 & 376 & 1199 & $\mathbf{8 0 9 2}$ \\
\hline \%PPerLoc/TPPerLM & 4,60 & 6,46 & 4,99 & 4,65 & 14,82 & $\mathbf{1 0 0 , 0 0}$ \\
\hline \%PPerLoc/PTELoc & 1,86 & 2,28 & $\mathbf{4 , 8 1}$ & 3,13 & $\mathbf{2 4 , 6 2}$ & $\mathbf{4 , 7 1}$ \\
\hline
\end{tabular}

Fuente: Elaboración propia sobre la base de información censal

La distribución para el único caso europeo entre estos cuatro países de nacimiento es completamente diferente respecto de los tres restantes. Los nacidos en Italia se concentran en el extremo norte del partido de La Matanza: se destacan Ramos Mejía y, en menor proporción, San Justo. En Ramos Mejía el peso de los nacidos en Italia es de casi un tercio del conjunto de los extranjeros, muy por encima del 9\% para el total del partido.

Finalmente, la población peruana se distribuye dentro del partido de una forma muy peculiar, con concentraciones coincidentes con las del conjunto boliviano en el extremo noreste del partido, aunque con valores absolutos y relativos mucho más modestos que aquellos. Se destaca Tapiales: un $24 \%$ de sus extranjeros son nacidos en Perú, frente al 4,7\% del total del partido.

Más allá de la diversidad de orígenes y distribución de las personas migrantes que estos datos muestran, en el próximo apartado identificaremos algunas iniciativas impulsadas desde el Estado a escala municipal vinculadas a las representaciones sobre la diversidad en un sentido amplio. Este análisis busca indagar en la vinculación entre políticas públicas y las particularidades de este extenso y complejo municipio.

\section{Las representaciones desde el Estado a escala municipal. Hacia una mayor complejización de la diversidad}

A continuación, se describen brevemente algunas de las políticas y programas de gestión estatal recientes a escala municipal que ponen el foco en las migraciones internacionales, y se analizan los supuestos sobre diversidad cultural que subyacen. El período bajo análisis se enfocó en los aos 2018 a 2020 dado el recorte temporal del proyecto marco de este trabajo. ${ }^{5}$ 


\section{a. 2018 - Antecedentes}

Se registraron algunas iniciativas en el marco de la "Plaza por los derechos de la niñez" el 27 y 28 de septiembre de 2018. Una de las actividades, denominada "Diversidad Cultural y Niñez", consistió en una mesa de disertación sobre "los procesos de integración cultural en la niñez y las políticas públicas”, en la que expusieron el Embajador de Bolivia en la Argentina, Santos Tito Véliz, y representantes de organizaciones por los derechos de niños/as y juventudes. También se desarrolló otra actividad, denominada "Diversidad Sexual e Identidad de Género en la niñez", a cargo de Gabriela Mansilla, presidenta de la Asociación Infancias Libres e integrante del panel Construcción de Identidad de Género en la Infancia en el marco de la Ley Nacional 26743.

Durante ese mismo año, circuló el afiche que se aprecia en la Figura 1, en conmemoración del Día del Respeto a la Diversidad Cultural, en el cual se identifican elementos propios de las culturas andinas y guaraníes (presencia de la bandera Whipala y atuendos folklóricos). Si bien parecen tener un objetivo de respeto, subyace una concepción de cultura de corte esencialista, que focaliza en ciertos atributos inmutables que devienen en la base de la relación nosotros/otros.

\section{Figura 1}

Efeméride Día del Respeto a la diversidad cultural

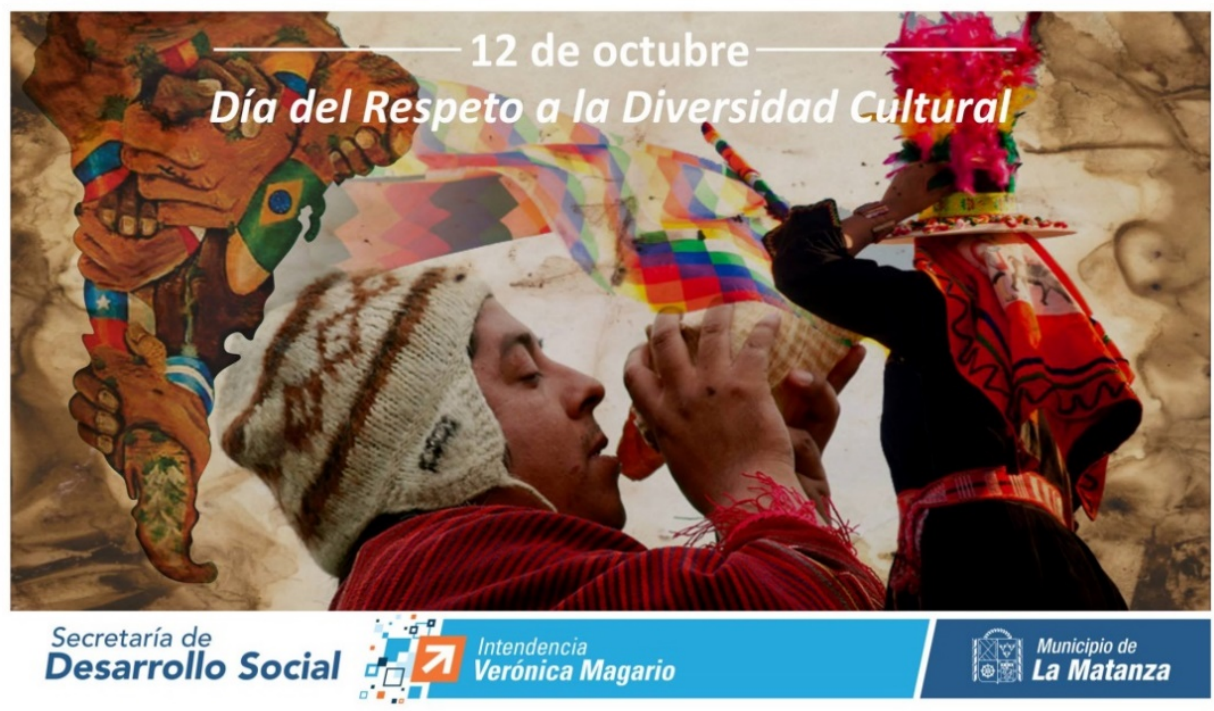

Fuente: Secretaría de Desarrollo Social, Municipalidad de La Matanza (2018)

Para esta misma fecha pero en 2020, y en el contexto de aislamiento por el Covid-19, la Secretaría de Cultura y Educación propuso un panel de discusión vía web (conversatorio): "América profunda. Aproximaciones a la cultura afro e indio americano", que se transmitió por la plataforma Zoom, del que participaron de afro argentinos/as del tronco colonial, guaraníes/paraguayos/ as y artistas plásticos y visuales, además de antropólogas y cientistas sociales. El conversatorio sostuvo una mirada transversal sobre género, diversidad y migraciones (afro, en este caso), y dio espacio a distintas voces representativas de la diversidad, evitando los clichés más folklorizantes y cosificadores que reducen la diversidad cultural a la etnia y a la nacionalidad. Ante la pregunta por los 
objetivos de la Secretaría respecto de la diversidad, un informante entrevistado afirma que ellos no son

(los purismos de los lenguajes sino pensar las políticas culturales desde una matriz más amplia y que la visión que se trabaja desde los entes estatales es la de un paternalismo, en el que hay "que ayudar", asistir a integrar a un Estado-nación que los contenga, que es una forma más atenuada de "civilizar", pero nunca considerarlos actores sociales activos que muchas veces, justamente, ponen en eje los límites del Estado-nación, y nos estimulan a replantearnos desde otros lados. Otras veces, también se busca estereotipar, esencializar las figuras. Espero que ese proceso empiece a nutrir la grilla de propuesta de actividades de los entes municipales, pero entendiendo que como punto de inicio es fundamental desarraigar la idea de que hay una sola "cultura" como una cosa homogénea y totalizante, como tampoco lo es dentro de la cuestión "indígena" o "afro" como algo que pervive único e inmutable, sino vivo y cambiante en sus múltiples acepciones. Creo fundamental, para saldar estos atropellos del Estado, que todos aquellos que lo componemos debamos trabajar más desde la necesidad y las propuestas de los actores de cada comunidad, y no generar proyectos de los cuales no se sientan parte ni sean actores principales de este cambio de paradigma (Entrevista a informante clave de la Secretaría de Cultura, 26/10/2020).

\title{
b. 2019 - Subsecretaría de Relaciones con Colectividades
}

En el mes de marzo de 2019 se crea la Subsecretaría de Relaciones con Colectividades. Al consultar por los objetivos de la Subsecretaría, se destacó que

\begin{abstract}
debido al gran crecimiento en la cantidad de vecinos inmigrantes con que cuenta nuestro partido, y a las necesidades y desprotección en la que se encontraban al llegar a un nuevo lugar para vivir, era necesario articular con ellos, y ayudarlos con todas sus necesidades. Sus instituciones y representantes barriales son el nexo con el municipio y todas las áreas correspondientes, Desarrollo Social, Cultura, deportes, protección ciudadana, así como todas las secretarías que forman parte del Estado municipal. (Entrevista a informante clave de la Subsecretaría 5/10/2020).
\end{abstract}

Entre las actividades de esta dependencia se destacan las desarrolladas con las colectividades de países vecinos:

a) La conmemoración de la independencia de la República del Paraguay (el 19 de mayo de 2019), celebrada en González Catán.

b) Acto en abril de 2019 con la participación del entonces presidente del Estado Plurinacional de Bolivia, Evo Morales, declarado Invitado Ilustre del Partido, en Lomas del Mirador.

c) Aniversario de la independencia boliviana (en agosto del mismo año), en Villa Celina.

En relación con colectividades europeas, se encontraba proyectada una actividad denominada "Matanza Celebra a Italia", que debió ser suspendida por la pandemia de Covid-19. También se encontraron efemérides de salutaciones por el aniversario de la Revolución de los Claveles el 25 de abril, fecha emblemática para la colectividad portuguesa, de gran visibilidad en Isidro Casanova.

Además de los intereses vinculados a las expresiones culturales, desde la Subsecretaría se señaló que también es tarea de la dependencia atender "a los vecinos de las colectividades (...) colaborando con ayuda de alimentos y distintos 
problemas que van surgiendo en el día a día". Se destaca, asimismo, el trabajo articulado para asistir a las instituciones junto con otras organizaciones del municipio (Asuntos Comunitarios), como también de otros niveles del Estado, como la Dirección Nacional de Migraciones, y oficinas consulares en el distrito de distintos países para tramitaciones de radicaciones y obtención de DNI. Cabe señalar que dentro de la jurisdicción se encuentran el Consulado de Paraguay y el Viceconsulado de Bolivia en San Justo, localidad cabecera del partido.

\section{c. 2020 - La creación de la Secretaría de Mujeres, politicas de género y diversidades}

El 3 de agosto se anunció en medios nacionales y locales la creación de la Secretaría de Mujeres, políticas de género y diversidades a cargo de Liliana Hendel, en un acto que contó con la presencia del intendente, de la exintendente y actual vicegobernadora Verónica Magario y de la Ministra de las Mujeres, Políticas de Género y Diversidad Sexual de la Provincia de Buenos Aires, Estela Díaz, junto a referentes del feminismo como Dora Barrancos. Durante el acto se hizo hincapié en la necesidad de que las políticas públicas tengan alcance territorial a través de la articulación entre los distintos niveles de gestión: "entender lo que significa la territorialidad, el lugar más cercano, el lugar donde vive la persona que sufre violencia, y ahí sabemos de la tarea incansable que nuestros intendentes y nuestras intendentas, y quienes están en esta trinchera, llevan adelante todos los días para abordar las necesidades de su pueblo" (discurso de Estela Díaz durante el acto). En palabras de Hendel: "si las leyes están lejos de la vida cotidiana de las personas, son letra muerta. Hoy venimos todas nosotras a hacer que esas decisiones de equidad e igualdad se conviertan en una realidad cotidiana. Estamos para recuperar la historia. La pandemia no nos detiene". ${ }^{6}$ De forma general, se pudo apreciar que las políticas de género exceden a la particularidad migratoria y que pareciera que las políticas migratorias no poseen una perspectiva de género manifiesta.

\section{d. Articulaciones proyectadas Nación - Municipio: delegación de la Dirección Nacional de Migraciones (DNM) y del Instituto Nacional contra la Discriminación, la Xenofobia y el Racismo (INADI)}

Durante el 2020 se hicieron visibles también las primeras iniciativas con miras a la creación de sedes/delegaciones de dos organismos de jerarquía nacional cuyo accionar se encuentra estrechamente vinculado a las migraciones y la promoción de la diversidad cultural.

En primer lugar, el 24 de junio, durante un recorrido del ministro del Interior Eduardo "Wado" de Pedro en el Mercado Central, ubicado en la localidad de Tapiales, se anunció la intención de ubicar en ese mismo sitio una dependencia de la Dirección Nacional de Migraciones. En palabras del ministro: “También visitamos el lugar en el que próximamente funcionará una oficina de la Dirección Nacional de Migraciones, que además de convertirse en la primera que funcionará en La Matanza, será un paso más para aumentar la articulación con el Gobierno Nacional". ${ }^{7}$ 
Por otro lado, el 4 de septiembre se anunció en las redes sociales del actual intendente Fernando Espinoza ${ }^{8}$ la apertura en La Matanza de una delegación del Instituto Nacional contra la Discriminación, la Xenofobia y el Racismo (INADI) para "fortalecer el trabajo que viene realizando en defensa de los derechos y de la igualdad”. En dicha comunicación se menciona la reunión con Victoria Donda, presidenta del organismo, dependiente del Ministerio de Justicia y Derechos Humanos de la Nación, y se afirma que desde esta futura delegación se buscará "generar promotores y capacitarlos para que vayan a cada una de las ciudades, a cada uno de los barrios de La Matanza a generar concientización; concientizar a nuestros chicos es poder tener la Argentina que queremos".

Como se mostró en este apartado, los esfuerzos desplegados desde el municipio por impulsar políticas públicas vinculadas con la diversidad, en un sentido amplio, han ido en aumento en los últimos años. En el próximo punto se avanza en los resultados de la encuesta con miras a identificar ciertas percepciones sobre la diversidad cultural en el municipio y contraponer estos resultados con el resto de las fuentes analizadas para este trabajo.

\section{Los resultados de la encuesta}

Entre las preguntas más directamente ligadas a la diversidad cultural, se indagó sobre cuáles eran las colectividades que, a los ojos del/a encuestado/a, tenían mayor presencia en el partido. Como resultado de esta pregunta, se puede decir que la colectividad boliviana es percibida como la de mayor presencia en el partido (fue mencionada en el $76 \%$ de las encuestas); en segundo lugar, la colectividad paraguaya (63\%) y en tercer lugar y cuarto lugar, las colectividades peruana y venezolana $(13,5 \%$ y $12,5 \%$, respectivamente).

En este punto vale reflexionar sobre dos aspectos. En primer lugar, se destaca la mayor percepción sobre la colectividad boliviana respecto de la paraguaya, cuando esta última es más relevante en términos cuantitativos. Esto podría dar cuenta de una mayor visibilidad de la comunidad boliviana, un aspecto que marca cómo operan las distancias entre las fuentes censales y las percepciones. Existen ciertos imaginarios racistas vinculados a la conformación de estereotipos racializados que circulan en la sociedad, y la colectividad boliviana es una de las más afectadas. Según el Mapa Nacional de la Discriminación del Instituto Nacional contra la Discriminación la Xenofobia y el Racismo (INADI) (2014), las personas migrantes de países limítrofes y del Perú son uno de los grupos más discriminados y que se percibe también como tal. Sólo para citar un ejemplo del año 2020, han circulado en los medios masivos de comunicación los mensajes racistas en Twitter del capitán del seleccionado argentino de rugby hacia la colectividad boliviana. ${ }^{9}$

Por otra parte, la colectividad boliviana en la Argentina cuenta con más de doscientas asociaciones civiles, políticas y culturales que son muy activas (Sassone, 2002; Pizarro, 2009; Melella, 2016). En La Matanza, se destacan los grupos bolivianos residentes en Ciudad Madero, Villa Celina, González Catán, Isidro Casanova y G. de Laferrere, donde se asientan varias asociaciones y se desarrollan festividades cívicas y religiosas, muchas de ellas en el espacio público. La presencia de la colectividad es muy fuerte a través de asociaciones como "Colectividad Boliviana Juana Azurduy de Padilla” y la "Asociación Vecinal” de Villa Madero. 
Villa Celina es el lugar más destacado por la cantidad de población del país andino que allí se concentra. También es notoria la cantidad de festividades que se celebran en todo el partido: alrededor de 18 al año. Entre ellas, se destacan la celebración de la Virgen de Copacabana (en 20 de Junio, Isidro Casanova, G. de Laferrere, González Catán, La Tablada, Rafael Castillo y Villa Celina), la Virgen de Urkupiña (González Catán y G. de Laferrere), Santa Vera Cruz (G. de Laferrere), Señor de la Cruz (Villa Celina) y El Señor de Maica (G. de Laferrere) (Agostino, 2007).

La colectividad paraguaya también posee gran cantidad de asociaciones: Avá Ka'aty Ne'é- Barrio Alberdi (González Catán), Asociación Guaireña de Deporte y Cultura (Rafael Castillo), Centro Amigos Guaireños de la Matanza (Isidro Casanova), Centro Carapegüeño y Damas Solidarias Carapegüeñas-Barrio Mi Esperanza (González Catán), Centro de Residentes Paraguayos de La Matanza (Ciudad Evita), Centro San Pedro de Paraná (Isidro Casanova) y Centro Silvio Morinigo (Isidro Casanova) (Agostino, 2007). Sus festividades son muy concurridas (aproximadamente 30.000 personas) pero se hallan acotadas a la celebración anual de la Virgen de Caacupé, el 8 de diciembre, en distintos lugares del municipio, entre los que se destacan el Club Deportivo Paraguayo en Villa Scasso, González Catán, y la Iglesia de Don Bosco ubicada en Ricchieri y Puente Doce.

Retomando los resultados de la encuesta, el segundo aspecto a considerar tiene relación con la antigüedad de los datos provenientes del Censo 2010, que impide contrastar con aquellas migraciones más recientes; especialmente con la venezolana, que marcó un punto de inflexión el año 2015 con un aumento en los pedidos de las radicaciones totales, que pasaron de 2.636 en 2014 a 70.531 en 2018 (Radicaciones, Dirección Nacional de Migraciones, 2018).

\section{Gráfico 1}

Países de los que provienen las colectividades que tienen mayor presencia en el partido según las personas encuestadas - Respuesta múltiple $(\mathrm{n}=201)$

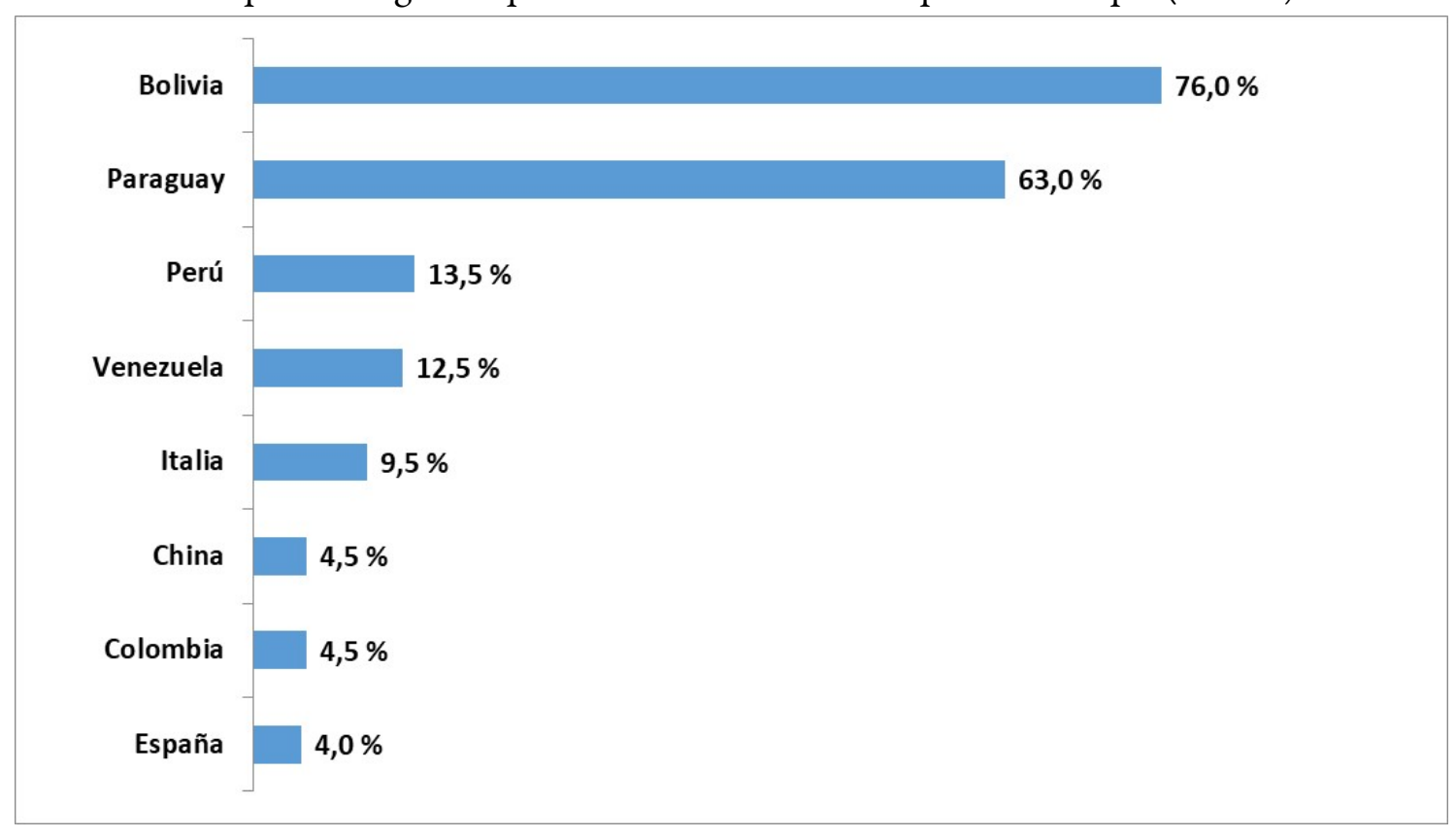

Fuente: Elaboración propia 
$\mathrm{Al}$ analizar estas respuestas considerando la localidad en la que fue tomada la encuesta (Tabla 3), estas percepciones parecen variar. Por ejemplo, en González Catán, si bien con poca diferencia, la colectividad paraguaya fue más mencionada que la boliviana y en la localidad de Ramos Mejía ambas colectividades fueron referidas por la misma cantidad de personas. En la localidad de San Justo, la colectividad italiana ocupa el tercer lugar (dato que no sorprende ya que allí se asientan algunas de sus asociaciones, como la Associazione Cattolica Cetrarese Benedetto y la Societá Italiana Stella Alpina de San Justo) y no la peruana ni la venezolana. Y en el caso de las localidades de Ramos Mejía y G. de Laferrere, la colectividad venezolana fue más mencionada que la peruana.

Retomando nuevamente el diálogo entre los datos de la encuesta y los registros del censo analizados en los apartados anteriores, resulta interesante dimensionar cómo ese protagonismo de la migración paraguaya en González Catán se replica tanto en las fuentes estadísticas como en las percepciones relevadas en esta localidad, sumado a la visibilización que adquiere a partir de su accionar en el espacio público, por ejemplo con las festividades religiosas como la Virgen de Caacupé.

Tabla 3

Países de los que provienen las colectividades que tienen mayor presencia en el partido según las personas encuestadas en cada localidad

\begin{tabular}{|c|c|c|c|c|c|c|}
\hline \multirow{2}{*}{ Países } & \multicolumn{5}{|c|}{ Punto de relevamiento } & \multirow{2}{*}{ Total } \\
\hline & San Justo & Tapiales & Ramos Mejía & Laferrere & González Catán & \\
\hline \multirow{2}{*}{ Paraguay } & 22 & 17 & 24 & 29 & 34 & 126 \\
\hline & $55,0 \%$ & $41,5 \%$ & $61,5 \%$ & $72,5 \%$ & $85,0 \%$ & \\
\hline \multirow{2}{*}{ Bolivia } & 29 & 32 & 24 & 35 & 32 & 152 \\
\hline & $72,5 \%$ & $78,0 \%$ & $61,5 \%$ & $87,5 \%$ & $80,0 \%$ & \\
\hline \multirow{2}{*}{ Italia } & 6 & 8 & 4 & 0 & 1 & 19 \\
\hline & $15,0 \%$ & $19,5 \%$ & $10,3 \%$ & $0,0 \%$ & $2,5 \%$ & \\
\hline \multirow{2}{*}{ Perú } & 4 & 9 & 7 & 2 & 5 & 27 \\
\hline & $10,0 \%$ & $22,0 \%$ & $17,9 \%$ & $5,0 \%$ & $12,5 \%$ & \\
\hline \multirow{2}{*}{ Venezuela } & 4 & 6 & 10 & 3 & 2 & 25 \\
\hline & $10,0 \%$ & $14,6 \%$ & $25,6 \%$ & $7,5 \%$ & $5,0 \%$ & \\
\hline \multirow{2}{*}{ Colombia } & 1 & 1 & 4 & 2 & 1 & 9 \\
\hline & $2,5 \%$ & $2,4 \%$ & $10,3 \%$ & $5,0 \%$ & $2,5 \%$ & \\
\hline \multirow{2}{*}{ Brasil } & 1 & 0 & 0 & 1 & 0 & 2 \\
\hline & $2,5 \%$ & $0,0 \%$ & $0,0 \%$ & $2,5 \%$ & $0,0 \%$ & \\
\hline \multirow{2}{*}{ China } & 1 & 4 & 3 & 0 & 1 & 9 \\
\hline & $2,5 \%$ & $9,8 \%$ & $7,7 \%$ & $0,0 \%$ & $2,5 \%$ & \\
\hline \multirow{2}{*}{ España } & 4 & 2 & 2 & 0 & 0 & 8 \\
\hline & $10,0 \%$ & $4,9 \%$ & $5,1 \%$ & $0,0 \%$ & $0,0 \%$ & \\
\hline
\end{tabular}

Fuente: Elaboración propia

Una pregunta fue ¿cuáles considera que son los modos en los que se expresa la diversidad cultural? Como respuestas más recurrentes se identificaron percepciones de carácter folklorizante: la comida regional fue mencionada en primer lugar (118 menciones), las fiestas regionales o patronales en segundo lugar (59 menciones) y la presencia de diferentes lenguas y modismos del habla en tercer lugar (51 menciones). 
Tabla 4

Modos en los que se expresa la diversidad cultural según los/as encuestados/as

\begin{tabular}{|c|c|c|}
\hline \multirow{2}{*}{ Expresiones de la diversidad cultural (Respuesta múltiple) } & \multicolumn{2}{|c|}{ Respuestas } \\
\hline & $\mathbf{n} \quad \mid$ & $\%$ \\
\hline Comida regional & 118 & $33,0 \%$ \\
\hline Las fiestas regionales/patronales & 59 & $16,5 \%$ \\
\hline Diferentes lenguas y modismos del habla & 51 & $14,2 \%$ \\
\hline Vestimentas típicas & 32 & $8,9 \%$ \\
\hline Las danzas típicas & 26 & $7,3 \%$ \\
\hline Características de personalidad atribuidas a las colectividades ${ }^{*}$ & 14 & $3,9 \%$ \\
\hline Artesanías & 13 & $3,6 \%$ \\
\hline Trabajo* & 13 & $3,6 \%$ \\
\hline Medicina tradicional o ancestral & 8 & $2,2 \%$ \\
\hline Música $^{*}$ & 8 & $2,2 \%$ \\
\hline Otros & 7 & $2,0 \%$ \\
\hline Ferias y centros culturales ${ }^{*}$ & 5 & $1,4 \%$ \\
\hline Reliqión ${ }^{\star}$ & 4 & $1,1 \%$ \\
\hline Total & 358 & $100,0 \%$ \\
\hline
\end{tabular}

Fuente: Elaboración propia

*Categorías creadas a partir del análisis de "otros"

Otra de las preguntas estuvo vinculada al conocimiento de festividades típicas de las colectividades, ya que se consideraron como indicadores de las percepciones de diversidad al ser prácticas de gran visibilidad para las colectividades de migrantes. Los resultados dan cuenta de cierto desconocimiento al respecto: como muestra el Gráfico 2, el 63\% (127) de las personas encuestadas dijo que "no" conocía ninguna festividad típica, y entre las personas que respondieron que "sí" conocían alguna, el 20\% (15) no recuerda cuál. Entre las respuestas de quienes pudieron mencionar explícitamente alguna festividad típica de las colectividades del partido, la más recurrente fue la fiesta de la Pachamama (22 menciones), en segundo lugar "la fiesta de la virgen" sin especificar cuál (7 menciones), y luego siguieron la Virgen de Copacabana (6 menciones), la Virgen de Urkupiña (5 menciones) y la Virgen de Caacupé ( 4 menciones), coincidiendo las últimas tres con las más representativas también para los migrantes. 


\section{Gráfico 2}

¿Conoce alguna festividad típica de esas colectividades?

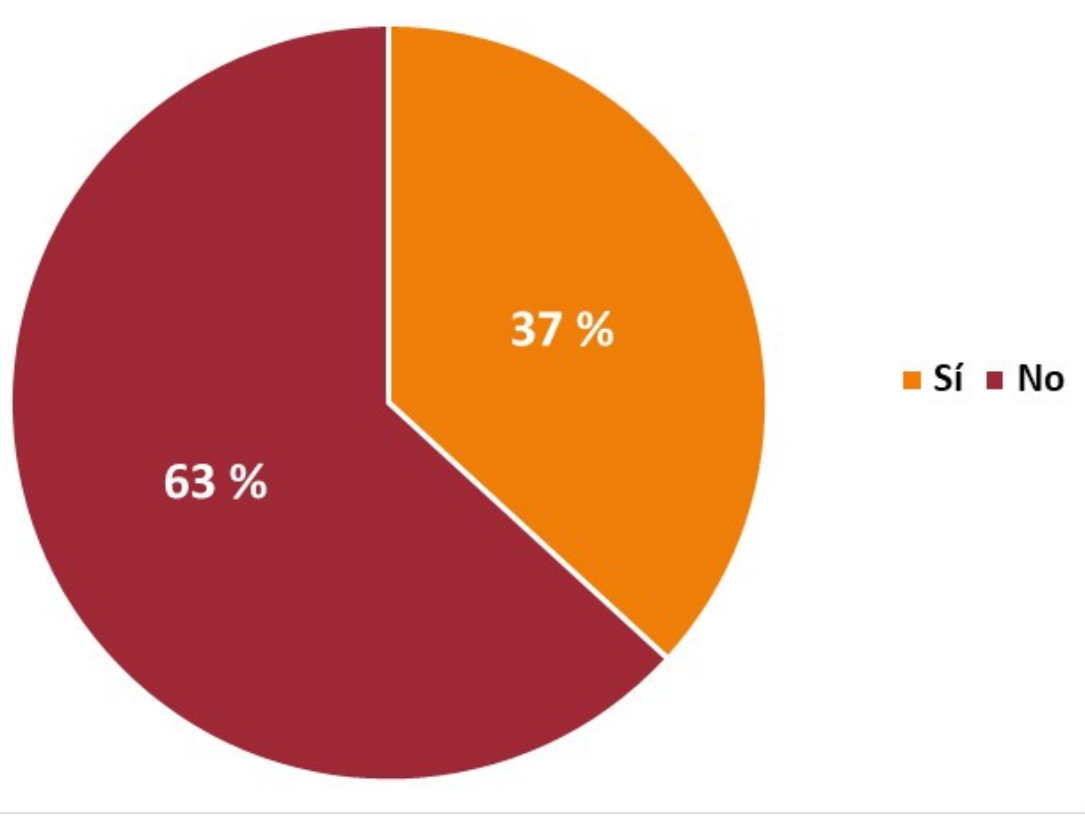

Fuente: Elaboración propia

Por último, se indagó sobre la percepción que las personas encuestadas tenían del rol del Estado en lo que respecta a la protección de la diversidad cultural. La mayoría (más del 70\%) considera que el Estado no protege o protege más o menos la diversidad cultural (Gráfico 3). Las respuestas a esta pregunta reflejan cierta percepción de desprotección a la diversidad cultural por parte del Estado, lo que podría eventualmente pensarse como un registro de demanda de políticas públicas específicas asociadas a esta temática. 


\section{Gráfico 3}

¿En qué medida el Estado protege la diversidad cultural?

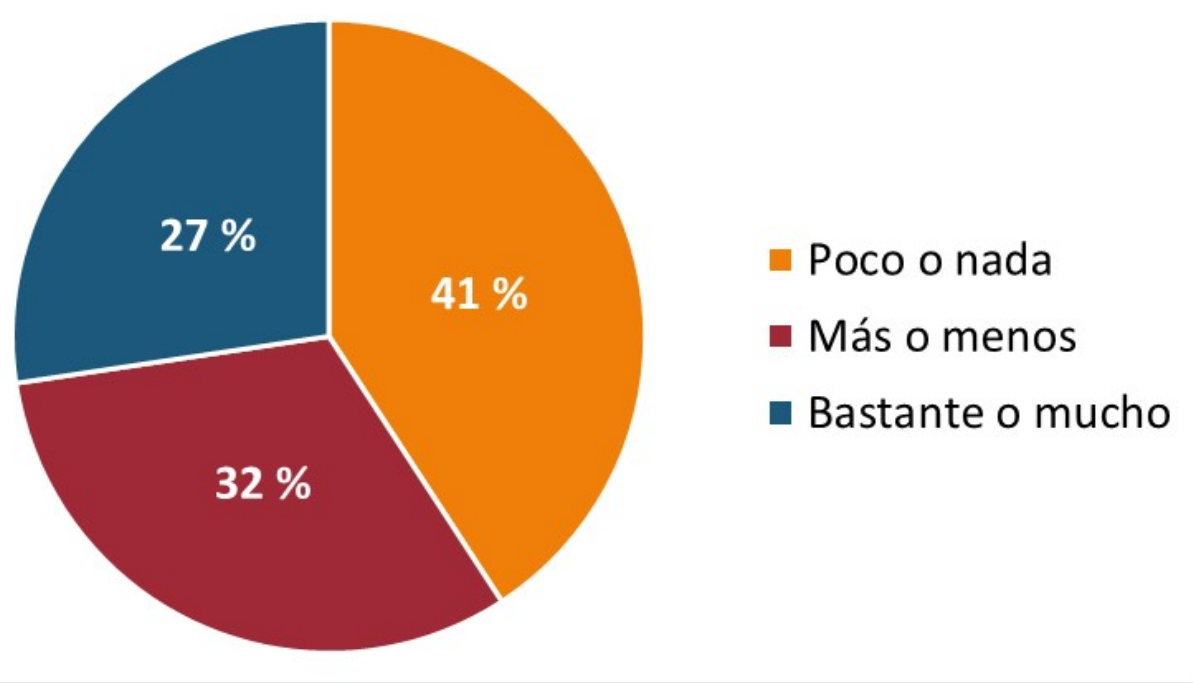

Fuente: Elaboración propia

\section{Reflexiones finales}

La Matanza se configura como un municipio en el cual el componente migratorio es relevante y diverso. Los orígenes de la población nacida en países distintos de la Argentina nos muestran cómo conviven en su interior procesos históricos de migraciones de ultramar con otros ya propios de la segunda mitad del siglo XX y de flujos recientes de principios del siglo XXI. Contraponiendo las fuentes censales con las obtenidas en la encuesta, se hallan por un lado puntos comunes que resaltan, por ejemplo, el protagonismo de la comunidad paraguaya en González Catán al tiempo que también se encontraron claros contrapuntos, como el sobredimensionamiento hacia la comunidad boliviana, nutrido por imaginarios racistas vinculados a la conformación de estereotipos racializados.

Respecto de las políticas públicas, pareciera que interculturalidad y diversidad cultural fueran sinónimos desde una mirada centrada en el Estado. Si bien se relevaron algunas actividades e iniciativas que tienden a la participación y transversalidad, pareciera que subyace una concepción un tanto monolítica de cultura que caracteriza con atributos de la diversidad que parecen existir previamente a la significación, en especial aquellos vinculados a la nación y a la etnia. Sin embargo, dado el carácter performativo de toda identificación, este escenario puede cambiar en la práctica y en la capacidad de acción. En este sentido, la perspectiva de género y su materialización en las Secretarías evidencia que el debate entre particularismos y generalizaciones en torno a la diversidad es actual y no cerrado en las esferas de gestión estatal. Iniciativas de esta clase pueden significar la apertura a un enfoque interseccional de la diversidad.

Otra intersección interesante se halla entre la percepción captada en la encuesta sobre cierta escasez sobre la protección que el Estado realiza de 
la diversidad cultural y el relativo impulso recientemente otorgado desde el municipio hacia las temáticas asociadas.

Respecto de los modos en los que se expresa la diversidad cultural, observamos que son frecuentes las representaciones de carácter folklorizante que se construyen sobre la posesión de rasgos esenciales y que se manifiestan en la gastronomía, las festividades y las lenguas. Sin embargo, consideramos que estas características, que a ojos de la academia adquieren un carácter estigmatizante, persisten en las representaciones populares, como fue identificado en la encuesta, razón por la cual pueden transformarse en recursos interesantes a la hora de gestionar desde una mirada transversal espacios de interculturalidad crítica. De esta manera, al reconocer la politicidad del término y las luchas, negociaciones y resistencias a partir de las que se define su significado, se evidencian su dinamismo e historicidad.

\section{Referencias}

Abba, A. (2005). Nuevas lógicas de centralidad urbana en el siglo XXI/ Área Metropolitana de Buenos Aires. Documento de trabajo CIHaM. Recuperado de: ht tp://bam21.org.ar/comunidad/pluginfile.php/1509/mod_data/content/5234/ Nuevas\%20L\%C3\%B3gicas\%20de\%20Centralidad\%20Urbana\%20\%20en\%20 el\%20S.\%20XXI.\%20Artemio\%20Abba.pdf.

Agostino, H. (2007). Los inmigrantes que eligieron vivir en La Matanza. San Justo: Universidad Nacional de La Matanza, Junta de estudios históricos, geográficos y estadísticos.

Ahmed, S. (2007). The language of diversity. Ethnic and Racial Studies, 30(2), 235-256.

Briones, C. et al. (2007). Escenas del multiculturalismo neoliberal. Una proyección desde el Sur. En A. Grimson (Comp.), Cultura y Neoliberalismo (pp. 265-299). Buenos Aires: CLACSO.

Devoto, F. (2004). Historia de la inmigración en la Argentina. Buenos Aires: Editorial Sudamericana.

Fraser, N. (2000). ¿De la redistribución al reconocimiento? Dilemas de la justicia en la era postsocialista. New left review, 0, 126-155.

Melella, C. (2016). Migrantes de paises andinos en la Argentina. Prensa gráfica, redes virtuales y construcción identitaria. Buenos Aires: Biblos.

Pacecca, M. I. y Courtis, C. (2008). Inmigración contemporánea en Argentina: dinámicas y políticas. Serie Población y Desarrollo, 84, 1-72.

Padilla, B., Azevedo, J. y A. Olmos-Alcaraz. (2014). Superdiversity and conviviality: exploring frameworks for doing ethnography in Southern European intercultural cities, Ethnic and Racial Studies, 38(4), 621-635. Recuperado de https://dx.doi.o $\mathrm{rg} / 10.1080 / 01419870.2015 .980294$.

Pizarro, C. (11 al 14 de junio de 2009). Organizaciones de inmigrantes bolivianos en áreas periurbanas argentinas: entre la demanda contra discriminación y la reproducción de la subalternidad. Trabajo presentado en Congreso de la Asociación de Estudios Latinoamericanos, Río de Janeiro. Recuperado de http://www.academia.edu/1203565/ORGANIZACIONES_DE_INMIGR ANTES_BOLIVIANOS_EN_AREAS_PERIURBANAS_ARGENTINAS_E NTRE_LA_DEMANDA_CONTRA_DISCRIMINACION_Y_LA_

República Argentina, Instituto Nacional de Estadística y Censos (2001). Censo Nacional De Población, Hogares y Viviendas. Recuperado 
de https://redatam.indec.gob.ar/argbin/RpWebEngine.exe/PortalAction?\&M ODE=MAIN\&BASE=CPV2001ARG\&MAIN=WebServerMain.inl\&_ga=2. 54137006.781714182.1535124060-510597371.1535124060

República Argentina, Instituto Nacional de Estadística y Censos (2010). Censo Nacional De Población, Hogares y Viviendas. Recuperado de https://redatam.indec.gob.ar/argbin/RpWebEngine.exe/PortalAction?\&M ODE $=$ MAIN\&BASE $=C P V 2010 B \& M A I N=$ WebServerMain.inl\&_ga $=2.536$ 88878.781714182.1535124060-510597371.1535124060

Sassone, S. M. (2002). Geografías de la exclusión, inmigración limitrofe indocumentada en la Argentina. Del sistema mundo al lugar (Tesis doctoral inédita). Facultad de Filosofía y Letras, Universidad Nacional de Cuyo, Mendoza, Argentina.

Sassone, S. y Matossian, B. (2014). Metropolización, migración y desigualdades sociales. Evidencias geográficas sobre la Región Metropolitana de Buenos Aires. En M. Di Virgilio y M. Pereleman (Coords.), Ciudades latinoamericanas. Desigualdad, segregación y tolerancia (pp. 221-252). Buenos Aires: CLACSO.

Svetlitza de Nemirovsky, A. y González, R. (1999). Saudade. La comunidad rural portuguesa de La Matanza. Argentina. Scripta Ethnologica, 21, 81-92.

Wright, S. (1998). La politización de la cultura. Anthropology today, 14(1), 1-19 [versión traducida por Florencia Enghel].

\section{Notas}

1 PICT 3166/2015 (2017 -2021) Migraciones, interculturalidad y territorio: Cartografías multiescalares de la inclusión social. Préstamo BID. Ministerio de Innovación, Ciencia y Tecnología. Agencia de Promoción Científica y Tecnológica. Dicho proyecto está radicado en el Instituto Multidisciplinario de Historia y Ciencias Humanas, Unidad Ejecutora CONICET, como Institución Beneficiaria (Buenos Aires, Argentina).

2 Cabe señalar que el concepto "extranjero/a", como "no nativo/a", es utilizado en este artículo en tanto categoría de análisis retomada de los propios relevamientos estadísticos, más precisamente de los censos nacionales, para indicar la población nacida en un país distinto de aquel donde está siendo censada. Se mantiene esta categoría de análisis al utilizar información censal, pero no es concebida en términos esencialistas, sino que su uso es resignificado en el reconocimiento de estos conceptos en tanto construcciones sociales vinculadas a la conformación de los Estados nacionales. Su empleo se enmarca en el aprovechamiento de la cobertura espacial y temporal que brindan los censos, aun con sus limitaciones y riesgos.

3 Desde la dimensión institucional y administrativa, el interior de La Matanza se configuraba, para 2010,en quince localidades: 20 de Junio, Aldo Bonzi, Ciudad Evita, González Catán, Gregorio de Laferrere, Isidro Casanova, La Tablada, Lomas del Mirador, Rafael Castillo, Ramos Mejía, San Justo, Tapiales, Villa Luzuriaga, Villa Madero y Virrey del Pino.

4 Nota: Las siglas de los indicadores utilizados se corresponden con TP: total población, Loc: Localidad, entre las principales.

5 En primer lugar cabe destacar, como marco macro, que la Constitución de la provincia de Buenos Aires, en su Artículo 11, contempla que la diversidad "(...) no admite distinciones, discriminaciones ni privilegios por razones de sexo, raza, religión, nacionalidad, lengua, ideología, opinión, enfermedades de riesgo, características físicas o cualquier otra condición amparada por las normas constitucionales”. También se encuentra presente en los artículos 39 (inciso 9), 44 y 200 (inciso 3).

6 "La Matanza creó la Secretaría de Mujeres, Políticas de Género y Diversidades y ya cuenta con el Fondo Especial de Asistencia en Violencias" en página web del Gobierno de la Provincia de Buenos Aires. Disponible en https://www.gba.gob.ar/mujeres/noticias/\%E2\%80\%9Cla_matanza_cre\%C3\%B 
3_la_secretar\%C3\%ADa_de_mujeres_pol\%C3\%ADticas_de_g\%C3\%A9nero_y_di versidades_y_ya

7 "Wado De Pedro recorrió el Mercado Central y destacó el 'abastecimiento de alimentos de las familias'” Agencia Nacional de Noticias - Telam 24/06/2020. Disponible en https://www.telam.com.ar/notas/202006/481221-eduardo-wado-depedro-mercado-central-alimentos.html

8 Nota publicada el 4/9/2020en la página de Facebook oficial de Fernando Espinoza. Disponible en https://www.facebook.com/147255748694051/videos/75724249174 2150

9 “(El capitán del seleccionado argentino de rugby, Matera) apuntó su odio hacia la comunidad boliviana. 'Sentarse en el asiento que apunta al revéz (sic) en el colectivo es lo más parecido a festejar tu cumpleaños en Bolivia con desconocidos", puso en uno de sus mensajes, mientras que luego insistió con otro supuesto chiste: 'Hombre boliviano porta mp3 con auriculares de ipod. Prueba suficiente para encarcelarlo por robo'”. Escándalo por los tuits racistas y discriminatorios del capitán de Los Pumas, Página / 12, $1^{\circ}$ de diciembre de 2020. Fuente: https://www.pagina12.com.ar/309030-escandalo-p or-los-tuits-racistas-y-discriminatorios-del-capi 